\title{
Flight Control Design Considerations for Multicopters Operating in Adverse Atmospheric Conditions
}

\author{
Augusto J. Zumarraga \\ CTA - Centro Tecnológico Aeroespacial \\ Facultad de Ingeniería - UNLP \\ La Plata, Argentina \\ uku@ing.unlp.edu.ar
}

\author{
Bernardo M. Concia \\ Departamento de Aeronáutica \\ Facultad de Ingeniería - UNLP \\ La Plata, Argentina \\ bernardo.concia@ing.unlp.edu.ar
}

\author{
Paul F. Puleston \\ Instituto de Investigaciones en Electrónica, Control \\ y Procesamiento de Señales (LEICI) \\ Facultad de Ingeniería, UNLP-CONICET \\ La Plata, Argentina \\ puleston@ing.unlp.edu.ar
}

\begin{abstract}
There is abundant literature related to the automatic control of multicopters showing the application of various control techniques, generally oriented to attitude control. This is one of the functions required for position and trajectory control, and is of greater academic interest due to its non-linear nature. However, it is not common to explore the conflicts that arise in the design when seeking to achieve high precision position control in adverse weather conditions.

In this paper we analyze objectives, structural limitations and implementation needs that significantly impact the performance of a control system under these conditions. Some design criteria are proposed and the aptitude of a linear control scheme is evaluated to respond to these requirements. The development efforts are also discussed to achieve the required precisions in the estimation of position for an application of these characteristics.
\end{abstract}

Index Terms-multicopter, flight control, LQG, atmospheric perturbation, integrated navigation

\section{INTRODUCCIÓN}

En años recientes la evolución de la propulsión eléctrica ha comenzado a impactar en el desarrollo de la aviación liviana. Los multicópteros no tripulados de pequeño tamaño ya se han consolidado como plataformas de observación aérea en una multiplicidad de usos; y ahora su evolución se orienta naturalmente hacia aplicaciones de mayor escala.

Es previsible que cuando se cuente con una nueva generación de celdas de almacenamiento de energía, los multicópteros eléctricos se conviertan en una opción viable para el transporte y distribución de cargas útiles, ya sea para logística o en aplicaciones más sofisticadas como la automatización de procesos de "pick and place" en la construcción de edificios; y seguramente evolucionarán hacia nuevas configuraciones para ofrecer soluciones como transporte personal en ambientes urbanos o sub-urbanos volando de forma autónoma.

Muchas de estas aplicaciones demandan alta precisión en el control de posición, y un elevado nivel de operatividad en relación a las condiciones climáticas. Nos proponemos analizar algunos aspectos a considerar para el diseño de un sistema de control capaz de responder a estas exigencias, tales como:

- precisiones alcanzables en el control de posición
- opciones de sensado con bajo costo

- naturaleza y modelado matemático de las perturbaciones

- aspectos a considerar en el modelado matemático de la dinámica del multirotor

- viabilidad de un esquema de control lineal para estas aplicaciones

En un multicóptero convencional el control de posición se logra variando el empuje neto, tanto en magnitud como en dirección. Lo primero se logra mediante una variación colectiva del empuje en todos los rotores; mientras que para lo segundo es necesario ajustar la orientación (actitud) del vehículo, operando sobre el empuje diferencial.

\section{Contexto Para el Diseño}

Las funciones principales del sistema de control de vuelo son el seguimiento de trayectorias con velocidad regulada, o bien el mantenimiento de la posición relativa respecto de un punto de interés (estacionario o no); dependiendo esto del escenario considerado.

En las fases de aproximación y ascenso, o tránsito en zonas congestionadas por obstáculos u otros vehículos se trata del primer caso; un vuelo de traslación en el cual el objetivo es mantener el vehículo dentro de una envolvente alrededor de determinada trayectoria, y recorrerla con velocidad regulada. En cambio, para el izado y entrega de cargas, despegue y aterrizaje en espacios reducidos, o como plataforma de trabajo para la manipulación de objetos en lugares de difícil acceso se espera que el sistema de control de vuelo garantice precisión en el alcance y mantenimiento de la posición relativa a un determinado objetivo; casos asociados al vuelo estacionario.

Para ofrecer un nivel de operatividad elevado en relación al clima, estos objetivos deberán ser alcanzables aun en condiciones de vientos fuerte.

\section{A. Notación y Ternas de Referencia}

En lo sucesivo haremos referencia a dos ternas cartesianas: una denominada geográfica local (terna- $l$ ) fija sobre la superficie terrestre, y otra solidaria al vehículo (terna- $b$ ), como se muestra en Fig.1. La posición $\boldsymbol{p}=\{x, y, z\}$ y velocidad 


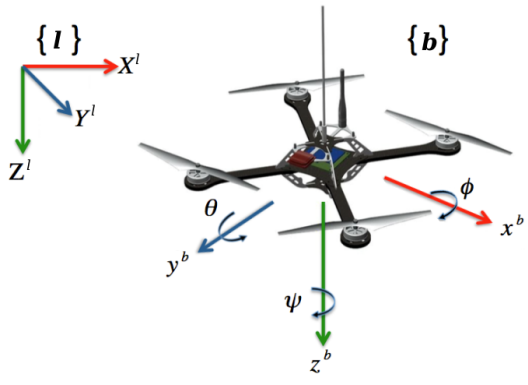

Fig. 1. Ternas de referencia.

$\boldsymbol{v}=\{u, v, w\}$ de la aeronave, así como la velocidad del viento $\boldsymbol{v}_{r}=\left\{u_{r}, v_{r}, w_{r}\right\}$, se proyectan en la terna local. La actitud de la aeronave se asocia a la rotación necesaria para llevar la terna- $l$ a la orientación de la terna- $b$, y se parametriza con ángulos de Euler $\boldsymbol{\theta}=\{\phi, \theta, \psi\}$ o mediante un cuaternión unitario $\boldsymbol{q}=\left\{q_{r}, q_{i}, q_{j}, q_{k}\right\}$. La velocidad de giro $\boldsymbol{\omega}=\{p, q, r\}$ se proyecta en la terna- $b$.

En las ecuaciones usaremos negritas cursivas para magnitudes vectoriales y negritas mayúsculas para operadores matriciales.

\section{B. Precisión y Sensado}

En primer término surge como pregunta de que forma se podría lograr la determinación de la posición relativa con alta precisión, sin depender de equipamiento especial externo.

Una aeronave que pretenda operar en ambientes no estructurados dependerá normalmente de algún sistema de navegación inercial; y dado que la posición y la actitud no son observables a partir de las mediciones inerciales, su determinación por integración diverge con el tiempo. Esto implica la necesidad de implementar algún esquema de fusión de datos para estabilizar la estimación, excepto que se cuente con sensores inerciales de muy bajo ruido y solo se consideren maniobras de poca duración en donde las divergencias dentro de las tolerancias admisibles.

1) Navegación Integrada Convencional: Existen opciones de bajo costo disponibles para satisfacer las necesidades de sensado para el control de vuelo. Los sensores inerciales MEMS llevan ya varios años en el campo de la navegación integrada para vehículos autónomos comerciales.

Es común utilizar un barómetro para estabilizar la estimación de altura (los hay de bajo costo con resoluciones del orden de $0.5 \mathrm{~m}$ ), y receptores GNSS para la determinación de posición y velocidad. Aunque la exactitud alcanzable por un GPS comercial es normalmente del orden de los $10 \mathrm{~m}$ y su tasa de actualización es baja, al dar información de posición global no se ve afectada por el movimiento de los obstáculos adyacentes. Pueden alcanzarse errores menores si solo interesa determinar desplazamientos acotados respecto de una referencia local durante tiempos cortos. Se puede lograr alta precisión con una estación en tierra para implementar un algoritmo diferencial.

Para medir posiciones relativas de forma directa existen sensores comerciales ultrasónicos con resolución milimétrica que resultan muy efectivos para distancias cortas (del orden de los $3 \mathrm{~m}$ ), pero su uso en exteriores queda condicionado por las características acústicas de las superficies en su entorno. Para distancias mayores y aptos para exteriores existen telémetros láser con resoluciones del orden de $1 \mathrm{~cm}$ y exactitud de $5 \mathrm{~cm}$ para rangos de hasta $50 \mathrm{~m}$.

2) Procesamiento de Imágenes: Mediante procesamiento de imágenes es posible complementar los esquemas anteriores para lograr alta precisión en la determinación de distancias relativas y velocidad de desplazamiento; sin requerir la asistencia de equipamiento adicional en tierra.

En la literatura se encuentran dos esquemas bien definidos y algunas variaciones: los métodos SLAM (Simultaneous Localization and Mapping) monocular o estéreo, y aquellos basados en el cálculo de flujo óptico. Los primeros dan una buena estimación de la localización y la actitud de la cámara pero son computacionalmente intensivos, dado que requieren construir un mapa global de todos los lugares visualizados. Los métodos basados en el cálculo de flujo óptico son computacionalmente livianos, pero solo determinan velocidad.

3) Algunos Valores de Referencia: A modo de ejemplo, los resultados en [1] señalan que es posible la determinación de posición con errores menores a $1 \mathrm{~m}$ volando a $6 \mathrm{~m}$ de altura mediante visión estereoscópica, mejorando los $3 \mathrm{~m}$ alcanzados en ese trabajo usando GPS. En [2] se reporta una precisión de $0.2 \mathrm{~m}$ para un helicóptero en escala (Rotomotion SR-100 UAV), mientras que en [3] se reportan errores con una desviación estándar de $0.055 \mathrm{~m}$ en el aterrizaje (T-Rex 600 ESP). Lamentablemente en estos trabajos no se indican las condiciones atmosféricas de los ensayos, y no hemos encontrado información adicional en relación a la precisión alcanzable en el control de posición para condiciones de viento fuerte.

\section{Perturbaciones}

Las perturbaciones que afectan la posición de una aeronave son de origen atmosférico. Toda aeronave es sensible a variaciones en su velocidad relativa respecto de la masa de aire que la rodea, tanto en magnitud como en dirección. El efecto resultante depende de su respuesta aerodinámica y de sus propiedades inerciales.

1) Respuesta Aerodinámica de un Multicóptero: Existen dos elementos básicos que aportan a la respuesta aerodinámica del multicóptero: la resistencia (o arrastre) de la estructura, y las fuerzas de sustentación de las palas de los rotores, que constituyen el efecto principal. Ningún fenómeno aerodinámico se desarrolla de forma instantánea, pero sus dinámicas son sub-amortiguadas y lo suficientemente rápidas como para aceptar la utilización de modelos cuasiestacionarios como aproximación.

a) Efectos en el fuselaje: La resistencia aerodinámica del fuselaje es una fuerza en la dirección del viento relativo $\boldsymbol{v}_{r}$, y proporcional al cuadrado de su magnitud:

$$
\boldsymbol{d}=-\frac{1}{2} \rho c_{D}(\alpha, \beta) \bar{S} \cdot\left|v_{r}\right|^{2} \hat{\boldsymbol{v}}_{r}
$$




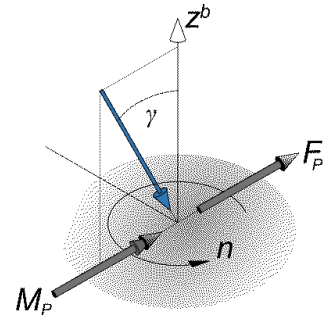

Fig. 2. Fuerza y Momento por flujo relativo no axial (efecto-P).

en donde $\rho$ es la densidad del aire y $\bar{S}$ es un área de referencia para adimensionalizar el coeficiente de resistencia $c_{D}$. Este depende de los ángulos $\alpha$ y $\beta$ que describen la dirección $\hat{\boldsymbol{v}}_{r}$ en la terna- $b$.

b) Efectos en el rotor: Los efectos aerodinámicos en un rotor son más complejos, porque el viento relativo se ve dominado por su régimen de giro. El efecto principal es la fuerza de tracción, que está alineada con el eje de giro. A partir de resultados experimentales [4] se observa que la tracción puede aproximarse en un rango amplio de condiciones aerodinámicas mediante la siguiente expresión:

$$
T=c_{n} n^{2}-c_{v} v_{T} n
$$

en donde $c_{n}$ y $c_{v}$ son constantes que dependen de la geometría de las palas, $n$ es el régimen de giro y $v_{T}$ es la componente de la velocidad relativa del aire en la dirección del eje de giro. Si además hay una componente normal al eje, se experimentan adicionalmente una fuerza y un momento alineados con dicha componente (ver Fig.2). Su magnitud se relaciona no-linealmente con el ángulo de ataque respecto del eje de giro, además de depender del régimen de giro del rotor. En aeronáutica esto se conoce como efecto- $P$.

Finalmente cabe agregar que por efectos aeroelásticos las palas se deforman, y con componentes de viento lateral esto provoca un cambio en el plano de giro, inclinándolo en la dirección del viento. Esto resulta en una fuerza de arrastre adicional a del efecto-P (ver [5]).

2) Modelos para la perturbación atmosférica: Habiendo modelado la respuesta aerodinámica es necesario ahora modelar las características de la perturbación atmosférica; es decir, del movimiento de la masa de aire en la cual se encuentra inmersa la aeronave. Este movimiento puede descomponerse en fluctuaciones lineales y rotacionales denominadas ráfagas, que se superponen a un desplazamiento medio cuasi-constante. Estas fluctuaciones en general son caóticas, y en tal caso colectivamente conforman lo que se denomina turbulencia.

Para diversos tipos de análisis las ráfagas pueden caracterizarse como variaciones estructuradas en la velocidad del aire a partir de modelos simples de cortantes de viento y ráfagas discretas; o como procesos estocásticos a partir de modelos para la densidad espectral de la energía de la turbulencia. De las opciones habituales para el modelo estocástico de turbulencia (ver [6]) se elige por simplicidad el de Dryden, recomendado en la MIL-F-8785C, que además provee modelos de distribución espectral para la componente
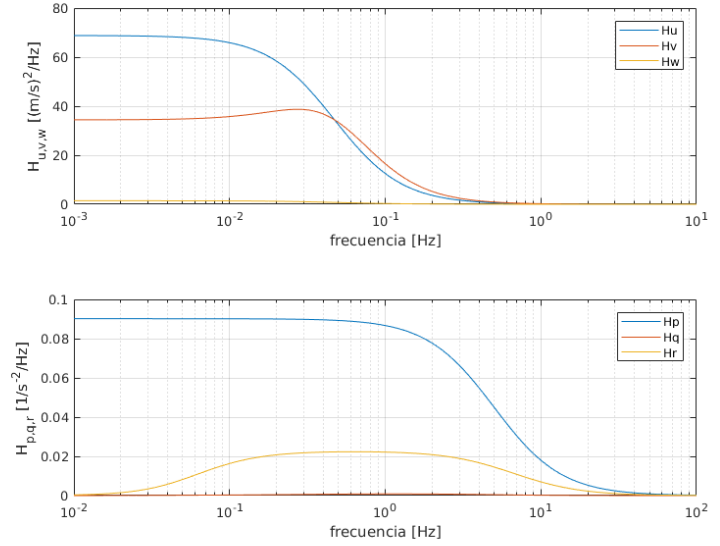

Fig. 3. Espectros para el modelo de Dryden con $U_{20}=30 \mathrm{knt}(\approx 15 \mathrm{~m} / \mathrm{s})$ a baja altura para velocidad lineal (arriba) y rotacional para una envergadura de $0.5 \mathrm{~m}$ (abajo). Puede observarse que la componente lineal vertical tiene energía mucho menor a las horizontales, mientras que para la parte rotacional se observan energías en frecuencias una década mayores y una componente pequeña para el eje $y$.

rotacional de la turbulencia (Fig.3). Puede notarse que la perturbación lineal, que afectará fundamentalmente al desplazamiento de la aeronave, está acotada a frecuencias de hasta $0.1 \mathrm{~Hz}$; mientras que la componente rotacional que afectará a la actitud posee una frecuencia de corte una década superior. Esto sugiere que en principio que sería necesario diseñar un lazos de control con un ancho de banda del orden de $0.5 \mathrm{~s}^{-1}$ para posición y $5 \mathrm{~s}^{-1}$ para actitud.

\section{Aspectos Centrales del Modelo Dinámico}

Idealizamos el cuadricóptero como un cuerpo rígido, y el modelo dinámico se descompone en uno de actitud y otro de desplazamiento.

\section{A. Dinámica de la Actitud}

Como se describe en [8], para la actitud el modelo puede obtenerse a partir de las ecuaciones cardinales de la mecánica para un cuerpo rígido:

$$
\mathbf{J} \frac{d \boldsymbol{\omega}}{d t}+\boldsymbol{\omega} \times(\mathbf{J} \boldsymbol{\omega}+\boldsymbol{h})=\boldsymbol{M}
$$

en donde $\mathbf{J}$ es el tensor de inercia, $\boldsymbol{h}$ es la suma del momento cinético de las masas rotantes, $\boldsymbol{M}$ es el momento resultante del empuje diferencial de los rotores respecto los ejes $x^{b}$ e $y^{b}$, junto con el torque reactivo de los rotores (oposición al torque en el eje de los motores) en el eje $z^{b}$. Los empujes están modelados con la ecuación (2), y por lo tanto dependen del viento relativo en cada rotor (donde incide $\boldsymbol{\omega}$ ) combinado con su régimen de giro, y del régimen de giro al cuadrado.

Físicamente el vector de control está constituido por los comandos para las velocidades de giro de los rotores, que para un cuadricóptero resulta de la forma $\boldsymbol{n}=\left\{n_{1}, n_{2}, n_{3}, n_{4}\right\}$. Las componentes de este vector aparecen en las tres filas de (3). Por ello lo habitual es definir acciones virtuales $\boldsymbol{\nu}=\left\{\nu_{x}, \nu_{y}, \nu_{z}, \nu_{t}\right\}$ como combinación lineal del vector real 
$\boldsymbol{\nu}=\mathbf{T}_{\nu} \boldsymbol{n}$. La matriz $\mathbf{T}_{\nu}$ se elije para que el control virtual se corresponda con los desbalances que definen los momentos en los ejes de la terna- $b$, junto con un comando de empuje colectivo $\left(\nu_{t}\right)$. En nuestro caso es:

$$
\mathbf{T}_{\nu}=\left[\begin{array}{rrrr}
1 & -1 & -1 & 1 \\
1 & 1 & -1 & -1 \\
1 & -1 & 1 & -1 \\
1 & 1 & 1 & 1
\end{array}\right]
$$

Pero es necesario replicar esto para los valores cuadráticos de los regímenes de giro. Si $\hat{\boldsymbol{n}}=\left\{\begin{array}{llll}n_{1}^{2}, & n_{2}^{2}, & n_{3}^{2}, & n_{4}^{2}\end{array}\right\}$, la misma transformación lineal $\mathbf{T}_{\nu}$ también define acciones virtuales cuadráticas $\hat{\boldsymbol{\nu}}=\left\{\hat{\nu}_{x}, \hat{\nu}_{y}, \hat{\nu}_{z}, \hat{\nu}_{t}\right\}$. Pero de esta forma el vector $\boldsymbol{n}$ se reemplazaría por dos definiciones paralelas de acciones virtuales, lo cual debemos evitar. Desarrollando las expresiones cuadráticas y reordenando encontramos que:

$$
\hat{\boldsymbol{\nu}}=\left\{\begin{array}{c}
\hat{\nu_{x}} \\
\hat{\nu_{y}} \\
\hat{\nu_{z}} \\
\hat{\nu_{t}}
\end{array}\right\}=\frac{1}{2}\left\{\begin{array}{c}
\nu_{x} \nu_{t}+\nu_{y} \nu_{z} \\
\nu_{y} \nu_{t}+\nu_{z} \nu_{x} \\
\nu_{z} \nu_{t}+\nu_{x} \nu_{y} \\
\left(\nu_{x}^{2}+\nu_{y}^{2}+\nu_{z}^{2}+\nu_{t}^{2}\right) / 2
\end{array}\right\}
$$

de lo que puede deducirse que:

$$
\left\{\begin{array}{l}
\hat{\nu}_{x} \\
\hat{\nu}_{y} \\
\hat{\nu}_{z}
\end{array}\right\}=\frac{\nu_{t}}{2}\left\{\begin{array}{c}
\nu_{x} \\
\nu_{y} \\
\nu_{z}
\end{array}\right\}+\frac{\nu_{z}}{2}\left\{\begin{array}{c}
\nu_{y} \\
\nu_{x} \\
0
\end{array}\right\}+\frac{1}{2}\left\{\begin{array}{c}
0 \\
0 \\
\nu_{x} \nu_{y}
\end{array}\right\}
$$

Por lo tanto se pueden expresar los valores cuadráticos en términos del vector virtual $\nu$, que por conveniencia adimensionalizamos en base al régimen de giro nominal $n_{0}$ para vuelo estacionario, adoptando: $\boldsymbol{\eta}=\boldsymbol{\nu} / 4 n_{0}$.

Incorporando 6 a lo desarrollado en [8] se puede condensar el modelo en la forma:

$$
\begin{aligned}
\dot{\boldsymbol{\omega}} & =\mathbf{F}(r, \boldsymbol{\eta}) \boldsymbol{\omega}+\mathbf{G}\left(\eta_{t}, v_{w_{z}}^{b}\right) \boldsymbol{\eta}+\boldsymbol{h} \eta_{x} \eta_{y}+\boldsymbol{d} \dot{\eta}_{z} \\
\dot{\boldsymbol{\theta}} & =\mathbf{C}(\boldsymbol{\theta}) \boldsymbol{\omega}
\end{aligned}
$$

en donde $v_{w_{z}}^{b}$ es la componente de la velocidad de viento en el eje $z^{b}$. La matriz dinámica $\mathbf{F}$ incluye amortiguamientos proporcionales al empuje colectivo $\eta_{t}$ en su diagonal, términos anti-simétricos de acoplamiento inercial y términos de acoplamiento aerodinámico entre cabeceo y rolido que dependen del comando de guiñada $\eta_{z}$. Con velocidad y comando de guiñada nulos, $\mathbf{F}$ es diagonal. La matriz de entrada $\mathbf{G}$ es casi diagonal, solo aparece un acoplamiento débil entre rolido y cabeceo también proporcional a $\eta_{z}$.

Los valores de $\eta_{t}$ dependen del control de posición, que en general tiene un ancho de banda menor al del control de actitud. Para el comando virtual $\eta_{z}$ del eje de guiñada $\left(z^{b}\right)$ puede decirse algo similar, ya que puede lograrse el apuntamiento del vector de empuje independientemente de él, y por lo tanto su regulación responde a requerimientos secundarios menos demandantes. El resultado es que en forma aproximada la actitud respecto del horizonte es lineal en el control $\left\{\eta_{x}, \eta_{y}\right\}$ utilizado para apuntar el vector de empuje.

\section{B. Desplazamiento}

Para el modelo de desplazamiento, en virtud de la escala de tiempos involucrada, es lícito considerar a la terna- $l$ como inercial. El vehículo se idealiza como una masa puntal sometida a la acción de un campo gravitatorio $\boldsymbol{g}$, a una fuerza de arrastre que resulta de la composición de la resistencia aerodinámica de la estructura $\boldsymbol{d}()$, un término $\boldsymbol{p}()$ equivalente a la suma del efecto-P en los rotores y el efecto aeroelástico; y el vector de empuje resultante de los rotores $\boldsymbol{f}()$ que depende de la actitud $\boldsymbol{\theta}$ y del comando de empuje colectivo $\eta_{t}$ :

$$
m \frac{d \boldsymbol{v}}{d t}=\boldsymbol{d}\left(\boldsymbol{v}_{r}\right)+\boldsymbol{p}\left(\boldsymbol{v}_{r}\right)+m \boldsymbol{g}+\boldsymbol{f}\left(\boldsymbol{\theta}, \eta_{t}\right)
$$

\section{Actuadores}

En algunos trabajos, en el modelado de multicópteros se opta por despreciar la dinámica de los actuadores (conjuntos rotor + motor + controlador) en cuanto a la relación entre comando y régimen de giro; pero esto no resulta aceptable en el caso de una aplicación de alto desempeño. En [11] se muestran resultados experimentales que evidencian un buen ajuste con un modelo de primer orden para la función de transferencia entre comando y velocidad de giro del rotor; con una constante de tiempo de 0.16 s para un rotor de 18 ". A pesar de las diferencias, para un rotor de 10 " hemos obtenido resultados similares en ensayos preliminares, identificando una constante de tiempo de $0.12 \mathrm{~s}$ con un retardo de $0.03 \mathrm{~s}$. Si bien el diámetro es mucho menor, también lo es el torque máximo del motor.

Si se asume que los lazos de control de posición y guiñada (rotación en $z^{b}$ ) tienen respuestas más lentas que las de actitud respecto del horizonte, se observa que las acciones virtuales de control para la actitud tendrán aproximadamente la misma dinámica que los actuadores:

$$
\dot{\boldsymbol{\eta}}=\omega_{m}(\boldsymbol{\mu}-\boldsymbol{\eta})
$$

donde $\omega_{m}$ es la inversa de la constante de tiempo de los motores y $\boldsymbol{\mu}$ es un vector de comandos virtuales para los controladores electrónicos de velocidad de los motores (ESC).

\section{Control Lineal}

Se pretende ahora evaluar la factibilidad de alcanzar alta precisión en la posición con un control lineal. En [8] concluimos que este permitiría alcanzar estabilidad robusta en la actitud para el modelo no lineal; aunque debemos advertir que en ese análisis no se incluyó la dinámica de los actuadores. Por otra parte, la masiva aplicación comercial del control lineal muestra que, mediante compensadores PID adecuadamente sintonizados, se pueden lograr resultados aceptables para el uso habitual. La búsqueda apunta a determinar la capacidad de rechazo de perturbaciones alcanzable.

En el siguiente planteo asumimos que se cuenta con un esquema adecuado para la determinación de la posición relativa respecto de un punto objetivo con precisión adecuada (en el orden del centímetro). En ese sentido planeamos el uso de un esquema de navegación integrada asistido por un sensor de flujo óptico de acuerdo con [9], [10], y medición 
de posición relativa a partir del seguimiento de "puntos singulares" (features) entre una imagen testigo tomada al inicio del posicionamiento y la actual utilizando un algoritmo ORB [7] (este trabajo se encuentra aun en desarrollo).

Para linealizar los modelos se considera como referencia una condición de equilibrio, lo que implica que $\dot{\boldsymbol{v}}=0, \boldsymbol{\omega}=$ 0 ; es decir, un vuelo estacionario o con desplazamiento a velocidad constante. En tal caso el modelo de actitud dado por (7) y (8) se convierte en un sistema lineal desacoplado en los ejes de la terna- $b$. Para una de las componentes $j:\{x, y, z\}$ de la velocidad angular $\omega$ el modelo resulta de la forma:

$$
\dot{\omega}_{j}=-a_{j} \omega_{j}+a_{j} \omega_{w_{j}}+b_{\omega} \eta_{j} \quad, \quad \dot{\theta}_{j}=\omega_{j}
$$

en donde $\theta_{j}$ equivale a una pequeña rotación en el eje $j$ de la terna- $b, \mathrm{y} \omega_{w_{j}}$ es la velocidad de rotación propia de la masa de aire.

Para el modelo de desplazamiento dado por (9) se sustituye la suma de los términos $\boldsymbol{d}$ y $\boldsymbol{p}$ por una expresión lineal con la velocidad $\boldsymbol{v}_{r}$, obteniéndose un modelo desacoplado para cada eje de la terna- $l$, con un término aerodinámico de amortiguamiento y sensibilidad a la ráfaga:

$$
\dot{v}_{i}=-d_{i} v+d_{i} v_{w_{i}}+b_{i} f_{i}^{l} \quad, \quad \dot{p}_{i}=v_{i}
$$

donde $v_{w_{i}}$ es la velocidad de ráfaga en el eje $i$ de la terna- $l$, y $f_{i}^{l}$ es la proyección del empuje en dicha dirección. Para el eje $z$ es necesario agregar además la aceleración gravitatoria $g$ como perturbación constante.

\section{A. Especificaciones de Diseño}

El objetivo es alcanzar la precisión requerida en la posición bajo condiciones atmosféricas adversas utilizando instrumentación de bajo costo. Inicialmente traduciremos esto a operar con tolerancias menores a $0.1 \mathrm{~m}$ en condiciones de viento fuerte $(35 \mathrm{knt})$ con un cuadricóptero comercial. Se toma como caso de estudio el de un "frame" DJI F450, para el cual se estiman para la actitud los siguientes parámetros: $a_{x, y} \approx-4.2, b_{x, y} \approx 500, \omega_{m} \approx 8.3$; mientras que para desplazamiento: $a_{x, y} \approx-0.12, a_{z} \approx-0.31, b_{x, y, z} \approx 0.87$.

\section{B. Control de Actitud}

Planteamos una síntesis LQG para control de actitud en los ejes $x^{b}$ e $y^{b}$ a partir de (11), aumentada con la dinámica de primer orden para el actuador. Para este regulador $\theta_{j}$ no es un valor absoluto de actitud, sino una rotación relativa requerida por el control de posición para orientar el vector de empuje. Por lo tanto esta variable no necesita ser estimada y se puede plantear el observador en base al siguiente modelo reducido:

$$
\begin{aligned}
\left\{\begin{array}{l}
\dot{\omega} \\
\dot{\eta}
\end{array}\right\} & =\left[\begin{array}{cc}
-4 & 500 \\
0 & -7
\end{array}\right]\left\{\begin{array}{l}
\omega \\
\eta
\end{array}\right\}+\left[\begin{array}{l}
0 \\
7
\end{array}\right]\{u\}+\left[\begin{array}{ll}
4 & 0 \\
0 & 1
\end{array}\right]\left\{\begin{array}{l}
w_{\omega} \\
w_{\eta}
\end{array}\right\} \\
y & =\omega+\nu
\end{aligned}
$$

Para el regulador se agrega a este modelo la relación $\dot{\theta}_{j}=\omega_{j}$. Discretizando el modelo con un tiempo de muestreo de $t s=$ $0.01 s$, y luego de algunas iteraciones sobre los parámetros del índice de desempeño se llega, con $\mathbf{Q}=\operatorname{diag}\left\{\begin{array}{lll}1 & 0.0025 & 0\end{array}\right\}$ y $\mathbf{R}=1$, a una matriz de realimentación de estados

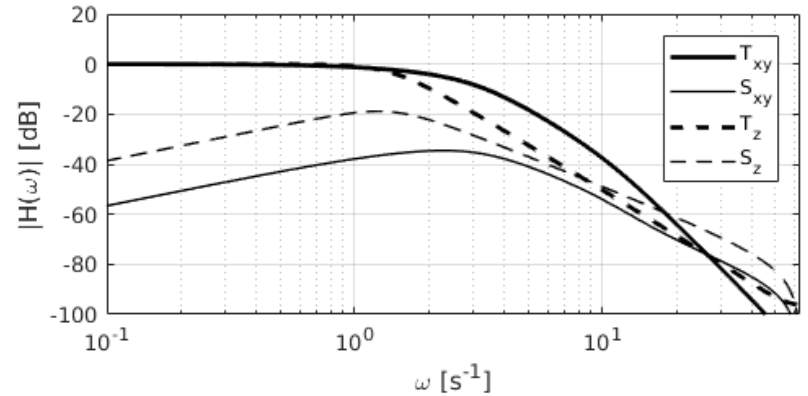

Fig. 4. Respuesta de lazo cerrado para posición horizontal $T_{x y}$ y vertical $T_{z}$, junto con las correspondientes sensibilidades a la perturbación atmosférica $S_{x y}$ y vertical $S_{z}$

$\mathbf{K}=\left[\begin{array}{lll}0.874 & 0.126 & 3.013\end{array}\right]$ que arroja una respuesta bien amortiguada y con un uso razonable del esfuerzo de control. Para el observador se considera el ruido de medición típico de un sensor de velocidad angular MEMS $\left(0.15^{\circ} / \mathrm{s} \mathrm{rms}\right)$ y un ruido de proceso con covarianza $\mathbf{W}=\operatorname{diag}\left\{10^{-5} 10^{-5}\right\}$, que es seleccionado también de forma iterativa para alcanzar una dinámica en el error de estimación algo más rápida que la del lazo de actitud. El lazo LQG resultante tendría un ancho de banda del orden de $11 s^{-1}$ para la referencia de actitud.

\section{Control de Posición}

Para el control de posición utilizamos la ecuación (12) y un modelo para dinámica de la fuerza de control $f_{i}^{l}$. Para el desplazamiento en el plano $x y^{l}$ este modelo debe representar la dinámica dominante del lazo de actitud, que en este caso puede ajustarse razonablemente con un modelo de segundo orden de frecuencia natural de $11 s^{-1}$ y amortiguamiento 0.8 . Para el eje $z^{l}$ se utiliza un modelo de primer orden con frecuencia de $8 s^{-1}$ correspondiente a la dinámica de los motores. Al vector de estado se agrega además la integral del error de posición en cada eje para rechazar las componentes constantes de las perturbaciones.

El sensado de posición depende del sistema de navegación integrada, por lo cual solo se plantea un regulador LQR. Para el plano $x y^{l}$, con un tiempo de muestreo $t s=0.2 s$ y matrices $\mathbf{Q}=\operatorname{diag}\{1 \quad 0 \quad 0 \quad 0 \quad 3\}$ y $\mathbf{R}=1$ se obtiene una matriz de

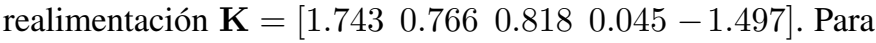
el eje $z^{l}$ se realiza un ajuste con $\mathbf{Q}=\operatorname{diag}\left\{\begin{array}{llll}1 & 10 & 1000 & 100\end{array}\right\}$ y $\mathbf{R}=1$, lo que arroja una matriz $\mathbf{K}=\left[\begin{array}{ll}1.919 & 1.4083 .785-\end{array}\right.$ 1.301] resultando en un menor ancho de banda. Esto último resulta necesario para acotar las variaciones del empuje colectivo, a fin de evitar saturaciones e interferencias con el control de actitud. La dinámica de lazo cerrado obtenida con estos ajustes se muestra en Fig.4.

\section{Simulación de Alta Fidelidad}

El esquema de control lineal en cascada se pone a prueba mediante una simulación de mayor fidelidad a la que ofrecen los modelos utilizados para la síntesis. Para ello, utilizando Matlab/Simulink ${ }^{\mathrm{TM}}$, se integran las ecuaciones cardinales de la mecánica con un esquema Runge-Kutta de orden 3 y tiempo de integración de $0.1 \mathrm{~ms}$. Para la respuesta aerodinámica de 

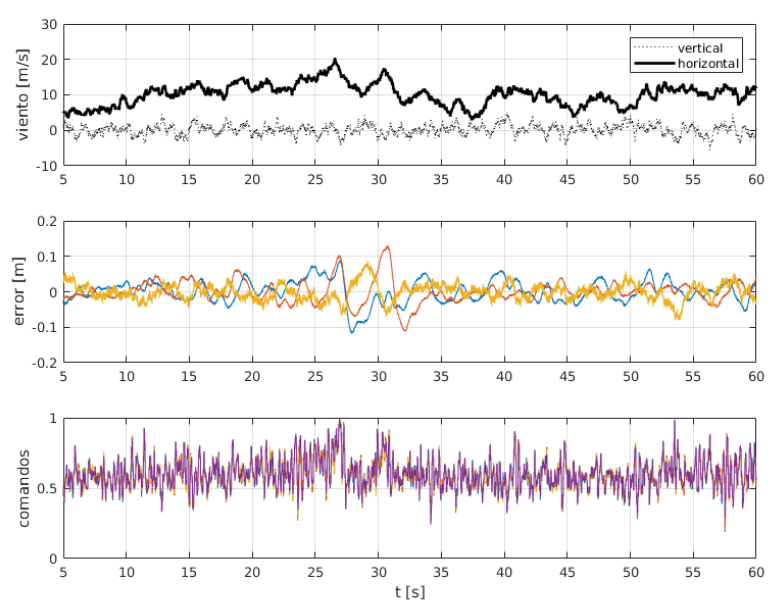

Fig. 5. Resultados de la simulación con modelo no-lineal, mostrando los errores de posición en vuelo estacionario a $2.5 \mathrm{~m}$ de altura, con viento medio de $30 \mathrm{knt}$ (a $6 \mathrm{~m}$ de altura).

los rotores se usan tablas obtenidas con métodos numéricos (elementos de pala) para diferentes valores de la velocidad de giro y del flujo relativo axial. Las velocidades de giro para cada rotor surgen de un modelo mecánico y un modelo no lineal del ESC, ajustado con los resultados experimentales. Se incluyen también los retardos, frecuencias y la cuantización de la etapa digital. Para la perturbación atmosférica se utiliza el modelo de Dryden para las seis componentes de la turbulencia, y un modelo potencial para la variación de la velocidad media del viento con la altura.

Se incluyen modelos de ruido blanco para los sensores inerciales y de posición relativa. La determinación de actitud se realiza implementando en el código del de vuelo un algoritmo adaptado de navegación inercial (ver [12]). Las rotaciones de comando para el control de actitud se calculan proyectando la fuerza requerida para el control de posición $\boldsymbol{f}^{l}$ en la terna- $b$, y a partir de esto se calculan las rotaciones del plano $x y^{b}$ para alinear el eje $z^{b}$ con el vector $\boldsymbol{f}^{l}$ :

$$
\theta_{x}=\tan ^{-1} \frac{f_{y}^{b}}{-f_{z}^{b}} \quad, \quad \theta_{y}=\tan ^{-1} \frac{-f_{x}^{b}}{-f_{z}^{b}}
$$

En Fig.5 se muestran resultados para vuelo estacionario a 2.5 $\mathrm{m}$ de altura con viento medio de $30 \mathrm{knt}(15 \mathrm{~m} / \mathrm{s}$, a $6 \mathrm{~m}$ de altura). Se observan errores en la posición del orden de \pm 0.15 $\mathrm{m}$, pero con alta exigencia sobre el esfuerzo de control. Un reajuste del diseño para achicar los errores aumentando el ancho de banda presenta dificultades por cuestiones robustez de este esquema lineal, mostrando respuestas muy oscilatorias.

\section{Conclusiones y Trabajo Futuro}

En este trabajo se ha realizado un análisis de las perturbaciones a las cuales se vería sometido un multicóptero operando con viento; y se ha propuesto un esquema de control lineal en cascada para mantener una posición horizontal y vertical con alta precisión. Para ello se utilizó una síntesis
LQR/LQG sobre una aproximación lineal desacoplada de la dinámica. En este modelo resulta necesario incluir modelos para la dinámica de los actuadores en el control de actitud y altura, y un modelo de la dinámica dominante del lazo de actitud en el control de posición.

De los resultados obtenidos se concluye que sería factible controlar la posición de un cuadricóptero con viento fuerte (F7 en la escala de Beaufort) dentro de una banda de error del orden de los $0.2 \mathrm{~m}$; pero con una alta exigencia en el esfuerzo de control. El diseño debe apuntar a un ancho de banda del orden de los $10 \mathrm{~s}^{-1}$ para el control de actitud y $1.5 \mathrm{~s}^{-1}$ para el control de posición.

La determinación de posición relativa respecto de un punto objetivo con alta precisión es sin dudas un aspecto tecnológico insoslayable en este planteo. Hemos propuesto utilizar un esquema de navegación integrada asistido por un sensor óptico; el cual aun se encuentra en desarrollo.

Respecto de la síntesis del control, aunque podrían existir márgenes para mejorar el esquema lineal, nuestro trabajo futuro apuntará a obtener soluciones mas robustas mediante técnicas de control no lineal.

\section{AgRAdECIMIENTOS}

Este trabajo fue realizado con el apoyo de la Facultad de Ingeniería-UNLP, el CONICET y la ANPCyT.

\section{REFERENCIAS}

[1] D. Pebrianti, F. Kendoul, S. Azrad, W. Wang, K. Nonami, ”Autonomous hovering and landing of a quad-rotor micro aerial vehicle by means of on ground stereo vision system", Journal of System Design and Dynamics, 2010

[2] N. Kuntz, P. Oh, "Autonomous cargo transport system for an unmanned aerial vehicle, using visual servoing", Journal of Systemics, 2009

[3] P. Pounds, A. M. Dollar, "Towards grasping with a helicopter platform: landing accuracy and other challenges", Proceedings of the 2010 Australasian Conference on Robotics and Automation, 2009

[4] J. B. Brandt, "Small Scale Propeller Perfomence at Low Speeds", Graduate College of the University of Illinois at Urbana-Champaign, 2005

[5] H. Huang, G. Hoffmann, S. Waslander, C. Tomlin, "Aerodynamics and control of autonomous quadrotor helicopters in aggressive maneuvering", Proceedings of the 2009 IEEE International Conference on Robotics and Automation, 2009

[6] T. Burton, D. Sharpe, N. Jenkins, E. Bossanyi, Wind Energy Handbook, John Wiley \& Sons, Ltd, 2001

[7] E. Rublee, V. Rabaud, K. Konolige, G. R. Bradski, ”ORB: An efficient alternative to SIFT or SURF", International Conference on Computer Vision, 2011

[8] A. Zumarraga, "Análisis de los acoplamientos inerciales y aerodinámicos en la dinámica de la actitud de un cuadricóptero", XVII Workshop on Information Processing and Control (RPIC), 2017

[9] B. D. Lucas, T. Kanade, Takeo, "An iterative image registration technique with an application to stereo vision", 7th International Joint Conference on Artificial Intelligence, 1981

[10] C. Tomasi, T. Kanade, "Detection and tracking of point features", International Journal of Computer Vision, 1991

[11] M. Yoon, "A transfer function model of thrust dynamics for multirotor helicopters", International Journal of Engineering Research \& Technology, 2016

[12] M. España, Sistemas de Navegación Integrada con Aplicaciones, CONAE, 2016 